\title{
Exploring knowledge landscapes: A narrative inquiry of midwives' experiences of working in diverse settings in Ghana
}

\author{
Evelyn Asamoah Ampofo*1, Vera Caine ${ }^{2}$, Jean D. Clandinin ${ }^{3}$ \\ ${ }^{1}$ School of Nursing and Midwifery, University of Cape Coast, Ghana \\ ${ }^{2}$ Faculty of Nursing, University of Alberta, Canada \\ ${ }^{3}$ Faculty of Education, University of Alberta, Canada
}

Received: December 13, 2018

Accepted: April 8, 2019

Online Published: April 24, 2019

DOI: $10.5430 /$ jnep.v9n8p36

URL: https://doi.org/10.5430/jnep.v9n8p36

\begin{abstract}
Objective: This paper focuses on exploring the experiences of midwives in Ghana who have worked in diverse settings over time. It explores how midwives' personal experiences across time, place and in diverse contexts impact their care for women during childbirth. The paper describes the forms of knowledge held by midwives. It presents how the experiences of midwives reflect their professional and personal practical knowledge landscape.

Methods: Using narrative inquiry, the experiences of four midwives working in private maternity homes were explored. Being guided by the three-dimensional narrative inquiry space of temporality, sociality and place, and the concept of relational ethics, a meaningful relationship was built with participants over a period of five months. Several tape-recorded conversations were held with each participant, multiple other interactions were recorded as field notes and in a journal. Each tape-recorded conversation was transcribed and used to construct narrative accounts that reflected participants' experiences as lived and told. Interim narrative accounts were shared with participants to ensure that the accounts reflected their experiences. Analysis: To identify resonant threads across all four narrative accounts, each account was read multiple times with intentionality and with the research objectives in mind.

Results: Three distinct professional knowledge landscapes for midwives were identified. These were the professional knowledge landscape of working in rural communities, urban communities, and private maternity homes. Two concepts of knowledge: knowledge for midwives and midwives' knowledge, were identified on each of these professional knowledge landscapes.

Conclusions: Education of midwives should consciously take into consideration the different knowledge landscapes in which midwives in Ghana practice.
\end{abstract}

Key Words: Midwives, Landscape, Narrative inquiry, Knowledge landscape

\section{INTRODUCTION}

While there is a history of traditional birth attendants in Ghana, gradually, over several decades, trained midwives are taking over provision of prenatal, labour and delivery care. More midwifery schools have been established to train different categories of midwives such as certificate, diploma, and graduate midwives. Education on the importance of birthing under the supervision of skilled birth attendants has Ghana. 
intensified over the last decade and yielded positive results in some parts of the country. However, there remains nonutilization of skilled birth attendants in some areas due, in part, to regional inequalities. ${ }^{[1]}$ For example low education which is high in some regions in the country were noted to influence the non-use of skilled birth attendance. The least educated women are noted to deliver without the assistance of a skilled birth attendant. The same is true for regions with poor socioeconomic infrastructure and resources. ${ }^{[1]}$ Even with progress in increasing the number of trained birth attendants in sub-Saharan Africa, only $59 \%$ of births were attended by trained birth attendants between 2012 - 2017, as compared to over $68 \%$ to $99 \%$ in other WHO regions. ${ }^{\text {[2] }}$ Despite progress, maternal death rates remain unacceptably high with an estimated 319 deaths per 100,000 live births. ${ }^{[3]}$ Indeed maternal deaths recorded in Ghana for 2016 reflects an increase in the maternal mortality ratio between 2013 to 2015. ${ }^{[4]}$

To improve the skills of midwives to respond to the global agenda to improve maternal health and reduce maternal mortality, the scope of midwifery care has expanded to include lifesaving procedures, family planning, and abortion care. Through the Safe Motherhood Initiative introduced by the WHO in 1987, many midwives in Ghana received training to provide emergency obstetric care. ${ }^{[5]}$

Midwives work within diverse contexts in Ghana. Each context of practice comes with unique demands, expectations, and challenges depending on where midwifery is practiced. Similarly, each midwife brings to midwifery practice a set of values, beliefs and knowledge developed through personal and professional experiences. ${ }^{[6]}$ Reflecting on the different contexts for midwifery practice in Ghana raises wonders about the practice contexts of midwives. The focus of this study is on understanding the types of knowledge midwives carry within their professional practice as well as the diversity of contexts in which they work. Of particular interest is the knowledge that midwives hold and express in different contexts. Specifically, the study sought to narratively inquire into experiences of Ghanaian midwives who care for women during childbirth in different contexts; inquire into the experiences of midwives within the professional knowledge landscapes in which they practice, and the personal practical knowledge they hold; explore how midwives' personal experiences across time, place and in diverse contexts impact their care for women during childbirth; and describe the forms of knowledge midwives embody. This paper discusses the different professional knowledge landscapes in which Ghanaian midwives work as identified from the study. It also discussed the different forms of knowledge midwives develop and hold as they work within different professional

Published by Sciedu Press knowledge landscapes.

\subsection{Understanding contexts of practice as professional knowledge landscapes}

As the researcher sought ways of conceptualizing different practice contexts in which midwives in Ghana work, she (The use of "the researcher" throughout refers to the corresponding author.) became familiar with Connelly and Clandinin's ${ }^{[7]}$ research on teacher knowledge in Canadian school contexts. Their understandings of professional knowledge landscapes and personal practical knowledge ${ }^{[7-9]}$ offered possible ways for understanding what midwives know and how their knowledge is expressed in their day-to-day interactions with clients, colleague midwives, and other members of healthcare teams. Their conceptualization of knowledge, in terms of personal practical knowledge and professional knowledge landscapes with roots in Dewey's ${ }^{[10]}$ theory of experience, offered a framework for the development of a conceptualization of midwives' knowledge.

Connelly and Clandinin ${ }^{[11]}$ described teacher's personal practical knowledge as expressed in their professional knowledge landscapes. Personal practical knowledge, a term designed to capture the idea of experience in ways that allows them to talk about teachers as knowledgeable and knowing persons, is in each teacher's present mind and body and in the future plans and actions. Personal practical knowledge thus is knowledge that reflects the individual's prior knowledge and acknowledges the contextual nature of the knowledge. Personal practical knowledge is knowledge teachers generate through their experiences over time. It can be described as teacher knowledge, that is subjective, tacit, embodied, gathered through experience and narratively expressed in knowledge landscapes. According to Connelly and Clandinin, ${ }^{[9]}$ a landscape metaphor is an appropriate metaphor to use in talking about practice contexts in that it allows people to talk about space, place and time. The professional knowledge landscape, the context in which teachers express their personal practical knowledge, is also filled with knowledge for teachers that is objective, theoretical and directly observable. This knowledge is given to teachers through formal education, policies, and workshops.

\subsection{Personal knowledge of midwives through stories}

Thinking about the knowledge landscape of midwives in light of Clandinin and Connelly's work with teachers offers ways to understand midwives' knowledge. ${ }^{[12]}$ Research on teachers' personal practical knowledge and their professional knowledge landscape shaped the research puzzle for this study. As Clandinin and Connelly puzzled over what stories teachers knew and told, we puzzled over what stories 
midwives knew and told. ${ }^{[12]}$ How do their stories shape midwives' knowledge and practice as they interact with clients and other midwives? It is important to understand how the stories in the knowledge landscape of midwives who participated in this study have been shaped by their knowledge, and how their knowledge has been shaped by their experiences in diverse practice contexts.

\section{Methodology}

Narrative inquiry, a relational research methodology, is "the study of experience as stories" (p. 374) and is "a way of thinking about experience". ${ }^{[13]}$ Clandinin and Connelly's narrative view of experience builds from Dewey's view of experience. ${ }^{[10,14]}$ Dewey laid out two criteria of experience: continuity and interaction. ${ }^{[10]}$ The criteria of continuity is that each experience takes up experiences from the person's past, is enacted in present situations, and has an effect on an individual's future experiences. The criteria of interaction indicate that an experience is always an interaction between a person and context. For Dewey experience occurs in a situation.

Narrative inquiry, both a way to understand experience and a way to study experience, framed the inquiry into the experiences of midwives and their experiential knowledge and knowledge landscapes. ${ }^{[15]}$ Clandinin and Connelly's inquiry framework is within a metaphoric three-dimensional narrative inquiry space with dimensions of temporality (past, present and future), sociality (pointed inward toward personal feelings, hopes, desires, aesthetic reactions and moral dispositions of the inquirer and participant, as well as outward toward the unfolding contextual events as well as to the relationship between participant and inquirer), and place. ${ }^{[14]}$ Place is described by Connelly and Clandinin as "the specific concrete physical and topological boundaries of place or sequence of places where the inquiry and event took place" (p. 480). ${ }^{[13]}$ The three dimensional space served as a framework for this research.

\subsection{Engaging midwives for the study}

Four midwives between the ages of 54 and 66 years old, were engaged in the study for over a period of five months. The four chose the following pseudonyms -Happy, Anna, Martha, and Adjoa for the purposes of the inquiry. The sample size of four was deemed appropriate since each participant generated rich text so that if more participants were added it may have created a challenge for us to adequately story the experiences of all participants. Additionally, engaging with four midwives facilitated the building of trusted relationships characteristic of narrative inquiry. A lager sample size would have defeated this methodological criterion. According to
Sandelowki sample size in qualitative research should permit "by virtue of not being too large, the deep, case-oriented analysis that is a hallmark of all qualitative inquiry. This also results in, by virtue of not being too small, a new and richly textured understanding of experience" (p. 183). ${ }^{[16]}$

To commence the study, ethical clearance from University of Alberta Research Ethics Board and from Noguchi Memorial Institute for Medical Research in Ghana was obtained. As part of the recruitment process, a list of private maternity homes, including phone numbers and locations in the Western Region was obtained from the secretariat of the Ghana Registered Midwives Association. The researchers were interested in how midwives' experience over time and in different places translate to knowledge and influence the care they provide. Participants were therefore purposefully selected to ensure that they had vast experience and had worked in different settings. Most Ghanaian midwives working in private maternity homes in Ghana have previous experience of working in government facilities and in diverse context, their stories were thought to likely reflect the advantages or complexities of the different context that influence midwifery practice in Ghana. Each potential participant was contacted mainly through phone call to book an initial face-to-face appointment. Details of the research were provided during the first interaction with each potential participant as a way of inviting them to the study. after which they were given some time to decide to be part of the study. Following acceptance to participate, consent form was signed. The researcher negotiated appropriate ways of engaging each participant given their unique circumstances. Respectful and trustworthy relationships were consciously built between researcher and participants based on the ethics of everyday life through informal interactions with participants before commencement of conversations.

\subsection{Being in the field}

Field in narrative inquiry is the relational space created by researcher and participants. ${ }^{[13]}$ In this study participants were asked to tell stories of experiences in tape-recorded conversations which provided participants and researcher space to share their stories and to begin to co-compose field texts. ${ }^{[14]}$ Being mindful of participants' busy schedules, places and times for conversations with each midwife were carefully negotiated. Phone calls to participants were made to foster research relationships between participants and researcher and also to negotiate dates, places, and times for each meeting.

Participants were invited to bring photographs and/or artifacts that spoke to their experiences to conversations. These artifacts and photographs and the midwife's stories around the items were also field texts. Participants brought pictures, 
certificates and citations that accompanied awards. The researcher kept a journal in which her feelings, reflections, and activities throughout the inquiry were recorded. Field notes were written for all conversations and journal entries.

\subsection{From field to field texts}

Field texts include transcripts of conversations, field notes, artifacts such as photographs, artwork and, documents that triggered memories. ${ }^{[14,17]}$ Each tape-recorded conversation was listened to several times and transcribed verbatim. The researcher storied, alongside transcripts and other field texts, her experiences, thus bringing forth personal responses to stories that participants told. As narrative inquiry is a relational inquiry methodology, both participants' and researcher's experiences are under study. Being aware of the possibility of deconstructing stories, ${ }^{[18]}$ transcribed field texts were narratively inquired into with attention to temporality, place and sociality.

\subsection{Writing interim and final research texts}

Interim research texts link the research puzzle and final research texts, in that, interim research texts are written with the research puzzle in mind, paying attention to place, time and context as reflected in the field texts. ${ }^{[19]}$ Interim research texts are part of making meaning of field texts and are co-composed as narrative accounts. ${ }^{[19]}$ Narrative accounts were shared with participants. Participants requested that researcher read aloud the narrative accounts to them at a negotiated time and place. Participants responded by reemphasizing some experiences. Allowing participants to respond to interim research texts ensures their stories are told in ways that reflect who they are and are becoming. Composing narrative accounts within the three-dimensional narrative inquiry space was a first level of analysis. A second level of analysis involved reading across all four narrative accounts to discern narrative threads.

\section{FINDINGS AND DISCUSSION}

\subsection{Finding resonance across narrative accounts}

Each narrative account was purposefully read and reread to identify resonant threads across the accounts. Reading the narrative accounts, being cognisant of the uniqueness of each person's experiences and the insights that could be gained from each storied life. One key narrative thread woven across the experiences of all four participants was identified.

Finding the balance between writing final research texts to represent participants' voices and the uniqueness of their stories, while keeping in mind the wider audience for the research, is a source of tension. ${ }^{[14]}$ To overcome this tension, the final research texts were written bearing in mind

Published by Sciedu Press the uniqueness of each midwife's experience, the ethical considerations inherent in the study, and the research puzzle.

\subsection{A Dominant narrative thread: Shaping personal practical knowledge in different professional knowl- edge landscapes}

The researchers conceptualized midwives' knowledge in two ways: personal practical knowledge and professional knowledge. As participants shared their experiences, they told stories about different places and contexts in which they worked at different times in their careers. Drawing from their stories of experiences, the researchers conceptualized midwives' work environments as their professional knowledge landscapes. While place is part of a professional knowledge landscape, a professional knowledge landscape is more than place. This paper discusses different types of knowledge in these landscapes, different people, relationships, events, and things in the knowledge landscapes, and different ways that people, places and things are in relationship within the landscapes. Our purpose was to show the differences that exist in midwifery professional knowledge landscapes in Ghana and to show how each influences the experiences of midwifery practice, as well as the life of each midwife.

Participants had worked in rural, and urban settings as well as private maternity homes. In subsequent paragraphs three professional knowledge landscapes identified are discussed.

\subsubsection{Professional knowledge landscape in rural commu- nities}

Two categories of knowledge that dominate the professional knowledge landscape of midwives, knowledge for midwives and midwife knowledge, were identified. Knowledge for midwives describes the professional knowledge that is given to midwives through formal education, training workshops, and protocol guidelines. Professional knowledge is highly objective, explicit, and easily communicated. This type of knowledge is what Polanyi referred to as propositional knowledge or the "know that" type of knowledge. ${ }^{[20]}$ Midwife knowledge, as distinct from knowledge for midwives, refers to the personal practical knowledge of midwives, which is experiential, embodied, narrative knowledge. Personal practical knowledge of midwives is non-propositional and represents the "know how" of midwifery knowledge.

Both professional knowledge, that is, knowledge for midwives, and midwives' personal practical knowledge shape the rural professional knowledge landscape of midwives. In the rural professional knowledge landscape, personal practical knowledge of midwives was more dominant for various reasons. Firstly, midwives worked alone in most facilities. They were often responsible for the health of entire communities 
and solely had to take decisions during labour, especially in times of emergency or complications. Participants were sometimes confronted with situations that their professional training had not adequately prepared them for. In such instances, midwives relied on their personal practical knowledge which was shaped from their past experiences, and was tacit in nature.

Secondly, relationships in rural professional knowledge landscapes were unique and complex. Acceptance of midwives into rural communities, as indicated by participants, was not solely dependent on the decision by the regional or district health management team to send a midwife to meet identifiable health needs of a community. It also depended on the communities' readiness to accept the services of the midwife. From the narrative accounts, there was a strong community involvement in the relationships that developed in the professional knowledge landscape. Martha, like other participants, narrated how she had to consciously build relationships with an entire community in order to get clients to solicit her services. She told how an entire community refused to attend a clinic built for them until she reached out to them. She reached out to mothers and to the community. "I needed the community to collaborate with me so I had to think of how best to do that... I could be there for over one month with no deliveries. I wanted that to change so I had to do that to win them... I did community sensitization. It wasn't easy, hmm! I had to go into the community to talk with them over and over to convince them to attend the clinic." Martha's account introduces another dimension of midwifery relationships, that is, midwives needed different knowledge to handle multiple relationships in the rural professional knowledge landscape. Since their professional training had not prepared them in this regard, participants relied on their personal practical knowledge to negotiate such relationships.

Participants mentioned relationships they built with significant individuals such as men (husbands), opinion leaders (Nackabah priests of a religious sect dominant in the Western Region), and Traditional Birth Attendants (TBAs). Happy believes the involvement of key personalities like the Nackabah priests is more important in the villages. "Culture is still working and is important in midwifery work in the village. The women go to different places in some communities. Here they go to 'Nackabah' they are given all sorts of concoctions to take when in labour. Before they come to you, they will go for special prayers from the Nackabah priest. It is part of their culture. But when they come and there is a problem with the delivery then it is on you, the midwife, and they feel it is the midwife's fault..."
The concept of relationship has received the attention of researchers for several decades yet its promotion in practice has been challenged. ${ }^{[21,22]}$ Whereas studies have been done relating to the midwife-mother relationship, little was found regarding community collaboration for successful midwifery practice.

Professional knowledge as evidenced by each participant's account was developed through formal midwifery training and education. Training refers to the programmes, of fered mostly for practicing midwives, to teach specific skills needed to be competent and proficient in their midwifery jobs, and to update them on current innovations. These workshops and protocol guidelines are fuelled by research and policies. The term education is used in this paper to refer to the formal classroom experience that student midwives receive, which is mainly focused on theoretical knowledge.

All participants shared experiences of attending workshops and using protocol guidelines in similar ways. Happy underscored the importance of workshops and how difficult it was to attend as she worked in rural communities. "The workshops are very good but it is difficult to find time to attend these workshops. When you are under somebody you have to ask permission. ....... at a one-man-station I have to get someone to be there so I go." Participants acknowledged the importance of training workshops and their willingness to attend although attendance was a challenge as they worked alone. After attending many workshops with many certificates to show, Happy described benefits of the training workshops and how her experiences in the workshops added to her personal practical knowledge: "They have workshops on new things that are coming up so we (midwives) get to know... We only get to know the new things when we go for workshops." Happy, who now owns a maternity home, partly attributes her decision to set up her private practice to the professional knowledge she received through attending workshops. Anna, a beneficiary of many skills training workshops, commented, "The workshops are very important for we midwives but not everyone wants to attend. Midwifery things keep changing so if you don't take advantage you will be practicing with old knowledge." Martha and Adjoa spoke of how workshops they attended facilitated the care they provided for women during labour in profound ways. Their personal practical knowledge was enhanced by the professional knowledge for midwives they received at workshops.

Situating the importance of training workshops in the rural professional knowledge landscape, participants talked about how important workshops are to enhancing their personal practical knowledge. According to Martha, "The workshops help us to get to know the new innovations. It is a ma- 
jor opportunity for midwives to continue to learn.” Martha traced her success as a professional midwife to out-of-schooltraining workshops. Similarly, Adjoa noted, "It's not only in the classroom that we get (professional) knowledge. When we attend workshops, a whole lot is learned. The protocols and training workshops make your mind sharper. It provides information on what to do and so you act fast." Training workshops and using clinical protocols remain key to quality health care delivery irrespective of whether the professional knowledge landscape is situated in rural, urban, or private settings. Using clinical protocol guidelines allow health care providers to offer appropriate care and treatment with benefits to both provider and client. ${ }^{[23]}$

Participants described how protocols helped them to know the interventions to use when there were complications. Anna spoke about her work at a district hospital, "When I got to this hospital on my first day I thought there would be doctors. I was confronted with a case of eclampsia. I saw the woman having seizures, I quickly organized myself and prepared the regimen of Magnesium sulfate (MgSO4) and administered it as per the protocol."

Global efforts to reduce maternal and infant mortality increased the need for midwives to be equipped with lifesaving skills. Emergence of diseases such as Human Immunodeficiency Virus, the widened scope of midwifery practice, global policies and agendas as well as innovations in science and technology necessitated change in curricular content for midwives. Decades ago midwives were not mandated to carry out interventions such as vacuum extraction and manual removal of the placenta. Such mandates were implemented to avoid delays that could lead to death or serious morbidities. ${ }^{[24,25]}$ Additional professional knowledge and skills midwives receive through training increases their professional autonomy to practice.

Today midwifery is seen as an answer to challenges of meeting maternal and newborn care for women and newborns globally. ${ }^{[26]}$ The aforementioned trends have led to a shift in the professional knowledge of midwives to accommodate the explicit knowledge needed in meeting new challenges. Midwifery education, training workshops, and the use of protocol guidelines provide important professional knowledge for midwives. These feed into the professional knowledge landscape of midwives even in rural communities and bring standards and uniformity in professional midwifery knowledge and practice.

\subsubsection{Professional knowledge landscape in district and re- gional hospitals}

Midwifery has been associated with more efficient uses of resources and improved psychosocial outcomes when provided by trained, licensed, and regulated midwives. ${ }^{[27]}$ Knowledge for midwives in the professional knowledge landscape for urban and regional hospitals was similar to that in the rural professional knowledge landscape. Professional knowledge was obtained through formal education, training workshops, and protocol guidelines. Whereas the professional knowledge needed to practice midwifery is similar across the two professional knowledge landscapes discussed so far, there were differences in the level of utilization of knowledge for midwives. At the district and regional hospitals midwives work in collaboration with other health professionals.

Midwives in district and regional hospitals work with strict institutional policies, and are conscious of professional boundaries that follow from policies and disciplines of health sciences. The professional knowledge landscape is strongly shaped by multiple health professionals who live within the landscape. Midwives are less likely to intervene during complications if they can call in a doctor to intervene. Midwives in rural areas, in cases where a referral was not possible, perform some procedures such as manual removal of placenta, or vacuum extraction. However, if the need arises for similar procedures in district or regional hospitals, midwives call a doctor.

Participants indicated how even in district and regional hospitals, they rely on their personal practical knowledge without calling other specialists when confronted with complications in the absence of a doctor. They called forth their personal practical knowledge to manage such situations. However, in district and regional hospitals they usually elicited the assistance of doctors and colleague midwives, even when they could have acted on their own personal practical knowledge.

Apart from the availability of doctors, the presence of supervisors such as administrators, nurse/midwifery managers, and senior colleagues also reduced the expression of midwives' personal practical knowledge because supervisors expected strict adherence to protocols. Strict adherence to protocol guidelines and procedure manuals tended to restrict the innovation of midwives. ${ }^{[28]}$ They recorded fewer experiences where they drew on their personal practical knowledge in district and regional hospitals.

In the professional knowledge landscape of district and regional hospitals, midwives are aware of ways they can express their personal practical knowledge. Anna shared an experience where she used her personal practical knowledge to help a baby in distress while she waited for the doctor. The doctor, when he arrived, was surprised at the sudden improvement of the baby's condition but Anna could not tell him exactly what she did because she could not support her actions with empirical evidence. Anna explained, "While I was 
working in the hospital I had a woman who came in labour. On auscultation the fetal heart $(F H)$ sound was very weak; it was actually falling. The doctor could not be reached. I had devised a way of dealing with low FH while working in the village. No one taught me but I had good results all the times I used it. It was my own 'kusum' ("Kusum" is from a local dialect meaning rituals. It represents unconventional and unorthodox actions taken by midwives/nurses to provide care.). So in this case I gave oxygen as well in addition to my 'kusum'. Within a short time, the FH started to pick up and eventually was stabilized. As soon as the uterus was cut open the baby was brought out crying. The doctor said, 'didn't we say the fetus was in distress?' Then someone said, not to the hearing of the doctor though, that Anna had performed her magic again".

As the above account shows, even in the professional knowledge landscape in district and regional hospitals, midwives' personal practical knowledge is expressed when a physician is not available. In urban, district and regional hospitals, midwives' personal practical knowledge is expressed but this was not captured in their nurses'/midwives' notes and reports.

\subsubsection{Professional knowledge landscape in private mater- nity home settings}

Participants attributed their decisions to operate or manage private clinics to the knowledge they gained from training workshops as well as their personal practical knowledge gained from experiences of working in one-man-stations. Happy narrated "... That is why some of us decided to come and do something for our own self (private maternity home) so we can practice what we have learnt from the many training workshops. I can practice in the village to help. All of us cannot go to the big hospitals so the people that are here we can help them. It is that experience that has helped me to run my own clinic. I take it that this is what the village work gave me. I don't need anything else."

Happy explained her motivation for opening a private maternity home. "The private idea started when I was planning to come home on retirement. A lot of courses I took in midwifery, family planning, the workshops and my experience. Am I to come on retirement and put it under the table? I decided that I will open a private clinic and help people."

Participants shared similarities in the knowledge expressed in their practices. The professional knowledge landscape in private maternity homes is similar to rural professional knowledge landscapes. Midwives work alone, are responsible for their actions and relied on their personal practical knowledge, enhanced by training workshops and protocols. They expressed their personal practical knowledge without fear. Happy said, "We cannot tell the doctors everything that we may have done if it is not part of the protocol. Once everything works well and the woman and her baby are both fine, you don't have a problem." Their secret stories were mostly shared at association meetings during informal conversations. Participants spoke about how professional knowledge gained through workshops and protocols helped them work at private maternity homes. Martha spoke of the partograph, a managerial tool for the management of labour, as an important tool that every midwife must use irrespective of where she works. "There are some skills that are difficult to get if you are not a professional." The partograph is one skill that is lacking at places where attendants are not professional midwives. Martha said, "For example the use of the partograph. It is the midwife who understands and can use it. Most of the maternity homes do not use it. At my private maternity home, for example, I have started using the partograph. It is high time that every maternity home gets a midwife who will run 24 hours. The ethics of the profession needs to be guarded." Both knowledge for midwives and midwives' knowledge are part of the professional knowledge landscape of the private maternity homes.

\subsection{A closer look at midwives' knowledge: Personal practical knowledge}

Personal practical knowledge is a conceptualization of knowledge that brings together the work of philosophers and researchers such as Dewey, Polanyi, Elbaz, and Schoen. ${ }^{[20,29-32]}$ Connelly and Clandinin defined personal practical knowledge as "a moral, affective, and aesthetic way of knowing life's educational situations."[9] Connelly \& Clandinin asserts, "Teachers develop and use a special kind of knowledge. The knowledge is neither theoretical, in the sense of theories of learning, teaching and curriculum, nor merely practical in the sense of knowing children."[6] Personal practical knowledge is both theoretical and practical, neither subjective nor objective but intersubjective. Verloop and colleagues suggest personal practical knowledge comprises individual experiences, personal history, and personality differences. ${ }^{[33]}$

While personal practical knowledge is constructed through individual personal experiences, there are resonating threads across the storied accounts of participating midwives. Field texts indicated midwives expressed their personal practical knowledge more extensively in rural communities and in "one-man stations". Midwives worked alone in these settings and interacted with different people and drew on their experiential knowledge, built over time, and embodied in who they were as persons. They drew on their personal practical knowledge when confronted with situations, including those 
without clear-cut solutions.

Personal practical knowledge includes what Polanyi referred to as personal knowledge, rooted in tacit knowledge. ${ }^{[10,34]}$ Polanyi, discussing non-propositional knowledge, argued that tacit knowledge is knowledge that is difficult to transfer to another person through writing, demonstrating or verbalizing. ${ }^{[10]}$ Tacit knowledge includes skills, ideas, and experiences that people have but which are not codified and may not be easily expressed. ${ }^{[35]}$ Personal practical knowledge, which includes tacit knowledge, is shaped by, and expressed in, the professional knowledge landscapes of participant midwives working in rural communities and in some urban settings.

Martha described midwives' personal practical knowledge as coming from multiple sources and was explicit about the difficulty in sharing personal practical knowledge. She said, "As for the knowledge it is not from one source. Midwives get knowledge through many ways ... Sometimes you know, you know something but you just don't know how or when you knew it and it is difficult to teach someone." She linked personal practical knowledge to experience by saying, "Different situations in life will teach you things. It is not only from school... sometimes the source may not even be related to midwifery at all".

Tacit knowledge cannot be given in lectures or found in textbooks, databases or manuals. ${ }^{[36]}$ Such knowledge is embodied and can be shared in action during interactions and informal meetings of midwives. It can also be shared when students or newly qualified midwives work closely with experienced midwives in clinical areas as a form of apprenticeship or mentorship or in other forms of social interactions of midwives.

Adjoa talked about how some of these actions are kept among midwives. "There is a whole lot that we do as midwives but sometimes we are afraid to document them because it is difficult to explain it. So, it is not only in the classroom that we get knowledge.” Happy remarked, "when we (midwives) meet, we gain a lot of knowledge we are always discussing among ourselves... You see, so you also pick that, when you are experiencing something you will remember."

Participants also shared how their interactions with nonhealth professionals such as birthing mothers and traditional birth attendants (TBAs) shaped their personal practical knowledge. All participants had various interactions with TBAs during their work in the rural areas. Though the midwives identified significant gaps in the professional knowledge and practices of TBAs, they acknowledged their ability to provide psychological care for birthing women and build trusting relationships with their clients. Martha, for example, noted, "Sometimes we midwives get some knowledge from the people we interact with. Like the TBAs... I admired the way they talk and support a woman in labour." According to Happy, "you can learn from everyone even from your clients." Participants also saw their own childbirth experiences as a rich source of knowledge. Through such experiences they understood the process of childbirth more deeply and were able to care for their clients with a better understanding.

\section{Conclusion}

We understand the importance of the professional knowledge landscape for midwives, which shapes both knowledge for midwives and midwives' personal practical knowledge. Personal practical knowledge, midwife knowledge, in addition to knowledge for midwives, is also important in providing care for women in labour. Each midwife's personal practical knowledge forms the basis of her practice in different professional knowledge landscapes. Because midwives working in rural communities are not constantly under the scrutiny of policy makers, administrators, and other members of health teams, they were able to express their personal practical knowledge in this professional knowledge landscape. Because midwives working in rural communities do not have the option of calling on obstetricians and other professionals for interventions, they are constantly presented with opportunities to experience new situations and to enhance their personal practical knowledge.

Returning to our research puzzle of what experiences midwives who work in diverse context have and how their experiences reflect their professional knowledge landscapes and the personal practical knowledge, it is clear that participants' experiences were unique but similar. All four expressed multiple but similar types of knowledge in all three professional knowledge landscapes. Their personal experiences across time, with various people and in different places, shaped their professional lives. It is evident that midwives work in different professional knowledge landscapes. Within these different landscapes, midwives build different relationships and these relationships influence midwifery practice and the lives of midwives differently. The different types of knowledge, that is, knowledge for midwives and personal practical knowledge, were expressed and developed differently on the landscapes. Personal practical knowledge was observed to be more prominent in the professional knowledge landscapes of rural communities and private maternity homes although they were identified in all three knowledge landscapes. Though knowledge for midwives was identified in all three professional knowledge landscapes, it dominated the professional knowledge landscape of urban settings. 


\section{IMPLICATIONS FOR MIDWIFERY EDUCA- TION AND PRACTICE}

\section{Professional contexts understood as professional knowl- edge landscapes}

The identification of three distinct professional knowledge landscapes for midwives in Ghana has implications for midwifery education, practice and research. We argue that midwives' knowledge is shaped only in part by their training. This inquiry showed how midwives' past and ongoing experiences shape their personal practical knowledge. The influences of place, time, and social interactions on the knowledge midwives hold was made explicit by the experiences they shared. Furthermore, illuminating the different professional knowledge landscapes of midwives has implications for midwifery education, practice and research.

Firstly, it calls for refocusing the education of midwives to ensure that the preparation of midwives takes into consideration different professional knowledge landscapes. Different forms of interactions as well as different types of knowledge used within these landscapes should be discussed during midwifery education. Students should be intentionally exposed to different landscapes in order to help them understand how to draw on their personal practical knowledge to work effectively in different professional knowledge landscapes.

Secondly, curriculum content should reflect the uniqueness of each landscape and the different relationships that are necessary within different knowledge landscapes. Potential challenges experienced in each professional knowledge landscape, as well as the types of knowledge that midwives develop and use in each landscape, should be addressed. Interest in working in rural professional knowledge landscapes during training should be encouraged. Recognition of different professional knowledge landscapes is important for the placement/postings of practicing midwives. As midwives transition from one professional knowledge landscape to another, programmes of orientation are needed. Participants indicated how transitioning from one professional knowledge landscape to another was physically and emotionally challenging. What midwives learn in classrooms, outside school in different contexts, over time, and in diverse places, influences what they know, and how they practice.

Finally, contextual, institutional, and personal influences that shape midwives' knowledge were brought forth. The study identified different professional knowledge landscapes and described complexities, relationships, and knowledge associated with each. Considering that little work has been undertaken on midwives' professional knowledge landscape in Ghana, this inquiry is a basis for further research into each professional knowledge landscape and personal practical knowledge.

\section{CONFlicts OF InTEREST Disclosure}

The authors declare that there is no conflict of interest.

\section{REFERENCES}

[1] Asamoah BO, Agardh A, Cromley EK. Spatial Analysis of Skilled Birth Attendant Utilization in Ghana. Global Journal of Health Science. 2014; 6: 34552. https ://doi.org/10.5539/gjhs.v6n4p 117

[2] World Health Statistics. Monitoring Health for the SDGs. 2017.

[3] UNICEF. Trends in Maternal Mortality. 1990 to 2015. WHO, Geneva, 2015.

[4] Ghana Health Services Family Health Division Annual Report. 2016.

[5] Okiwelu T, Hussein J, Adjei S, et al. Safe motherhood in Ghana: Still on the Agenda? Health Policy. 2007; 84: 359-367. https: //doi.org/10.1016/j.healthpol.2007.05.012

[6] Asamoah Ampofo E. A Narrative Inquiry into the Experiences of Midwives in Ghana, Caring for Women during Labour. Unpublished doctoral dissertation, University of Alberta, Edmonton. Alberta, Canada, 2018.

[7] Connelly FM, Clandinin DJ. Personal practical knowledge and the modes of knowing: Relevance for teaching and learning. In E. Eisner (Ed.), Learning and teaching the ways of knowing. NSSE Yearbook. Chicago IL: University of Chicago Press; 1985; 174-198.

[8] Clandinin DJ. Terms for inquiry into teacher thinking: The place of practical knowledge and the Elbaz case. Journal of Curriculum Theorizing. 1985; 6: 131-148.
[9] Connelly FM, Clandinin DJ. Teachers as curriculum planners: Narratives of experience. New York: Teachers' College Press; 1988.

[10] Dewey J. Experience of education. In DJ. Clandinin (Ed.) Engaging in Narrative Inquiry. Chicago: Left Coast Press; 1938.

[11] Connelly FM, Clandinin DJ. Teachers' professional knowledge landscape. New York: Teachers' College Press; 1995.

[12] Clandinin DJ, Connelly FM. Teachers' Professional Knowledge Landscapes: Teacher Stories. Stories of Teachers. School Stories. Educational Researcher. 1996; 25: 24-30. https://doi.org/10 .3102/0013189X025003024

[13] Connelly FM, Clandinin DJ. Narrative inquiry. In J. L. Green, G. Camilli, \& P. Elmore (Eds.), Handbook of complementary methods in education research. 3rd ed. Mahwah, NJ: Lawrence Erlbaum, 2006.

[14] Clandinin DJ, Connelly FM. Narrative inquiry: Experience and story in qualitative research. San Francisco, CA: Jossey-Bass; 2000.

[15] Connelly FM, Clandinin DJ. Stories of experience and narrative inquiry. Educational Researcher. 1990; 19: 2-14. https ://doi.org/ 10.3102/0013189x019005002

[16] Sandelowski M. Focus on qualitative methods: Sample size in qualitative research. Research in Nursing \& Health. 1995; 18: 179-183. https : //doi.org/10.1002/nur. 4770180211

[17] Clandinin DJ. Engaging in narrative inquiry. Chicago: Left Coast Press; 2013. 
[18] Gergen M. Once upon a time. A narratologist's tale. In C. Daiute \& C. Lightfoot (Eds.), Narrative analysis: Studying the development of individuals in society. Thousand Oaks, CA: Sage; 2004; 267-285.

[19] Clandinin DJ, Caine V. Narrative Inquiry. In A. A. Trainor \& E. Graue (Eds.) Reviewing qualitative research in the social sciences. New York: Routledge; 2013; 166-179.

[20] Polanyi M. Personal knowledge towards a post-critical philosophy. London: Routledge and Kegan Paul; 1958.

[21] Berg M, Lundgren I. To support and strengthen caring in childbearing. Student litterature, Lund. 2004.

[22] Hunter LP. A hermeneutic phenomenological analysis of midwives' ways of knowing during childbirth. Midwifery. 2008; 24: 405-415. PMid:17889971 https://doi.org/10.1016/j.midw. 2007.06 .001

[23] Heymann T. Clinical protocols are key to quality health care delivery. International Journal of Health Care Quality Assurance. 1994; 7: 14-17. PMid:10140847 https://doi.org/10.1108/095268 69410074702

[24] Thaddeus S, Maine D. Too far to walk: Maternal mortality in context. Social Science and Medicine. 1994; 38: 1091-1110. https: //doi.org/10.1016/0277-9536(94)90226-7

[25] Barnes-Josiah D, Myntti C, Augustin A. The "Three Delays" as a framework for examining maternal mortality in Haiti. Social Science and Medicine. 1998; 46: 981-993. https://doi.org/10.1016/ S0277-9536 (97) 10018-1

[26] Sakala C, Newburn M. Meeting needs of childbearing women and newborn infants through strengthened midwifery. The Lancet Series. 2014: 384; 9948. https://doi.org/10.1016/S0140-6736(14 )60856-4
[27] Renfrew MJ, McFadden A, Bastos HM, et al. Midwifery and quality care: findings from a new evidence-informed framework for maternal and newborn care. Lancet. 2014; 384: 1129-45. https : //doi.org/10.1016/S0140-6736(14)60789-3

[28] Mahran MA, Paine M, Ewies AAA. Maternity guidelines: Aid or hindrance? Journal of Obstetric Gynaecology. 2007; 27: 774-80. https://doi.org/10.1080/01443610701667353

[29] Dewey J. Art as experience. New York: Capricorn Books. 1934.

[30] Elbaz F. The teacher's "practical knowledge": Report of a case study. Curriculum Inquiry. 1981; 11: 43-71. https : //doi .org/10.108 $0 / 03626784.1981 .11075237$

[31] Elbaz F. Teacher thinking: A study of practical knowledge. London: Croom Helm; 1983.

[32] Schoen D. The reflective practitioner: How professionals think in action. London: Temple Smith; 1983.

[33] Verloop N, Driel JV, Meijer PC. Teacher knowledge and the knowledge base of teaching. Journal of Educational Research. 2001; 35: 441-461. https://doi.org/10.1016/S0883-0355(02)0 0003-4

[34] Polanyi M. The tacit dimension. Chicago: The University of Chicago Press; 1966.

[35] Chugh R, Wibowo S, Ghandi S. Mandating the transfer of tacit knowledge in Australian Universities. Journal of Organizational Knowledge Management. 2015; 1-10.

[36] Haldin-Herrgard T. Difficulties in diffusion of tacit knowledge in organizations. Journal of Intellectual Capital. 2000; 1: 357-367. https://doi.org/10.1108/14691930010359252 